\title{
Aplicación de un ciclo de mejora en el aula a la asignatura "Generación de Energía Térmica" del Grado en Ingeniería de Tecnologias Industriales
}

\section{Application of an improvement cycle in classroom to the subject "Generation of Thermal Energy" of the Degree in Industrial Technology Engineering}

José MANUEL SALMERón LISSÉN

ORCID: http://orcid.org/0000-0003-4340-6564

Universidad de Sevilla

Departamento de Ingeniería Energética jms@us.es

Fecha de recepción: 03-06-2019.

Fecha de aceptación: 06-06-2019.

DOI: http://dx.doi.org/10.12795/9788447221912.008 Pp.: 202-224 
En este artículo se aplican los conocimientos adquiridos en un programa de mejora docente a una asignatura de corte tecnológico de tercer curso del Grado en Ingeniería de Tecnologías Industriales, y se hace hincapié en los aspectos metodológicos, de selección y organización de contenidos necesarios para la correcta impartición de la misma, y por último de los sistemas de evaluación que se pueden implementar para valorar e incluso motivar a los alumnos. Nuestra conclusión particular es que se debería extender la realización de este tipo de cursos para la mejora de la calidad docente en la universidad.

Palabras clave: Generación de Energía Térmica, Grado en Ingeniería de Tecnologías Industriales, Docencia Universitaria, Experimentación Docente Universitaria

\section{Abstract}

There are several professional improvement programs but with relatively little frequency university professors and lecturers consider the need to perform one. The accumulated experience, the prizes, acknowledgments, and a positive self-evaluation, may cause this situation. In this article the knowledge acquired in an improvement program for teachers is applied to a technological subject of the third year of the Degree in Industrial Technology Engineering, the emphasis is placed on the methodological aspects, selection and organization of contents, and finally on the evaluation systems that can be implemented to assess and even motivate the students. Our particular conclusion is that the completion of this type of course should be extended to improve the quality of teaching in the university.

Keywords: Thermal Energy Generation, Energy Engineering, University Teaching, Experimental University Teaching

Jornadas de Formación e Innovación Docente del Profesorado | № 2 (2019) Esta obra se distribuye con la licencia Creative Commons 


\section{Introducción}

Existen numerosos programas de mejora profesional, pero con relativamente poca frecuencia los profesores universitarios consideramos la necesidad de realizar uno de estos. La experiencia acumulada, los premios, reconocimientos, y una autoevaluación positiva, pueden ser algunos de los culpables de que se dé esta situación. Este artículo surge para resumir las iniciativas, aprendizajes y conclusiones aplicadas por el autor tras recibir un curso general de docencia universitaria y ponerlas en práctica en forma de ciclo de mejora en el aula (CIMA). La aplicación de un ciclo de estas características a una asignatura universitaria, cuando la experiencia docente del profesor es dilatada, es siempre un reto. Este reto tiene diversas vertientes, en lo personal porque de alguna manera el profesor considera que ya lo sabe todo con respecto a la docencia, más aún si por vocación y desde el comienzo de su carrera docente se ha preocupado de recibir formación, y si sabe que dicha formación ha surtido efecto por la obtención de algún premio o reconocimiento a su docencia. En la vertiente docente, porque de alguna manera el profesor considera que de esa asignatura ya se sabe lo que hay que impartir y lo que no, lo que encaja y lo que sale fuera del contexto de un curso determinado. En la vertiente científica, porque el profesor-investigador cree conocer todos los vericuetos de la asignatura. En este contexto, cuando aceptamos los numerosos restos anteriormente planteados lo hacemos asumiendo la revisión de numerosos aspectos y concepciones personales respecto a la docencia.

En este artículo se describe, según la experiencia personal del autor, el proceso seguido durante el diseño, la aplicación y la evaluación y valoración de un ciclo de mejora aplicado a la asignatura Generación de Energía Térmica del Grado en Ingeniería de Tecnologías Industriales, de la que el mismo es coordinador. En todos los momentos 
que se van a describir a lo largo del texto del artículo se van a considerar tres aspectos fundamentales de la docencia universitaria como son la metodología, los contenidos y los sistemas de evaluación.

Hay que tener presente que no es muy común encontrarse con ciclos de mejora docente aplicados a una asignatura técnica del área de la ingeniería. Se han consultado textos publicados en las Actas de las Jornadas de formación e innovación docente editadas por la Universidad de Sevilla. En dichas referencias puede verse que en gran medida se refieren a asignaturas de primer o segundo curso (Beltrán, 2017; Leñero, 2016) y/o de carácter no tecnológico o aplicado (Barbadilla-Martín, 2017; Pérez, 2016), en lugar de tercer curso y con un marcado componente tecnológico como es el objeto de este artículo. Artículos de los citados se centran en una parte de la asignatura o en un procedimiento muy concreto de la misma. Sin embargo, en este caso, pese a aplicar el ciclo de mejora a dos bloques temáticos, los razonamientos y conclusiones metodológicas, de contenidos y de evaluación obtenidos se refieren a la misma tratada como un todo. En cualquier caso, todas las referencias anteriormente citadas de asignaturas técnicas se han usado como punto de inicio de las actividades desarrolladas por el autor en los ciclos de mejora que ha implementado durante el curso de docencia universitaria (Programa FIDOP de la Universidad de Sevilla) y que son el objetivo del presente artículo.

\section{Diseño previo de los ciclos de mejora docente}

Como se comentó anteriormente en la introducción, el diseño previo de los ciclos de mejora abarcó tanto el modelo metodológico, como los contenidos y la evaluación. Este es el motivo de que se hable de ciclos de mejora en plural, ya que se trata de dos ciclos. En primer lugar, se diseñó un modelo metodológico viable y, posteriormente 
en el tiempo, transcurridas 5 semanas, se propuso un segundo ciclo en el que se mantuvo el modelo metodológico que se complementó con una adecuación de los contenidos de las siguientes 4 sesiones de clase.

\section{Modelo metodológico}

El modelo metodológico viable se diseñó durante la tercera semana de docencia del segundo cuatrimestre, por lo tanto, una vez que los alumnos ya habían comenzado la asignatura y el autor había aplicado la metodología docente que durante su experiencia ha ido considerando como la más adecuada, pero sin reflexionar sobre los aspectos de la misma, y sin el conocimiento de si existían teorías desarrolladas que aconsejasen una metodología sobre otra. De una reflexión profunda, basada tanto en lo recibido en clase por parte de la formadora, como por lo expresado por Rivero y Porlán (2017), se generó el modelo representado mediante el siguiente gráfico donde, PP: Planteamiento del problema o debate sobre un caso real; AC: Análisis crítico de los resultados del problema, y E: Problema similar al resuelto en la clase, propuesto a los alumnos para hacer en casa, que sirva de entrenamiento y no sea repetir exactamente lo ya hecho.

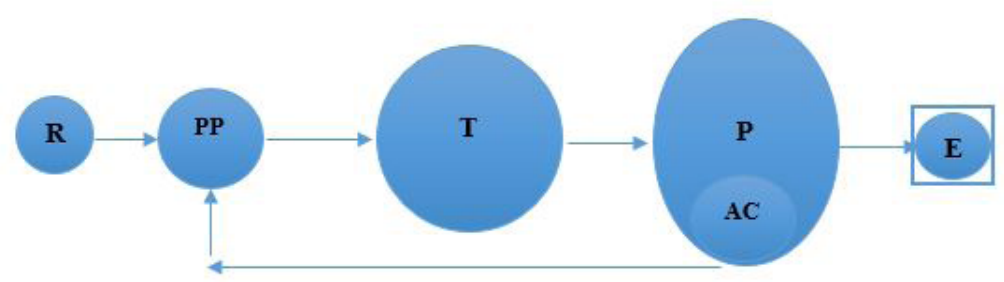

Figura 1. Modelo metodológico viable

Este modelo metodológico se aplicó a dos bloques temáticos de la asignatura. Por un lado, al bloque temático correspondiente a "Aerodinámica de llamas industriales" que se imparte en cuatro sesiones de una hora y veinte minutos cada una, y por otra, al bloque temático 
correspondiente a "Intercambio radiante en atmósferas de combustión" que se impartió 4 semanas después del anterior. Este dato es particularmente interesante porque el desarrollo del segundo modelo, mediante la planificación de la secuencia de actividades en las que se concreta, lo pude realizar con mayor facilidad en la segunda ocasión, de lo que se obtiene la conclusión de que la práctica de este tipo de planificación docente basada en una metodología es más fluida cuanto más se practica y por tanto cuanto más se extiende a la totalidad de la docencia. Por motivos de espacio, sólo hemos incluido en este artículo la secuencia de actividades correspondiente al segundo periodo de tiempo. Ambas experiencias se realizaron en el mismo grupo, formado por unos 40 alumnos, y en la misma localización, un aula convencional con bancas fijas al suelo.

A continuación, se incluyen las tablas donde se nombran las diferentes actividades, se describe su contenido, se explica en qué consiste cada una y se añade el tiempo estimado para su realización práctica.

Tabla 1. Secuencia de actividades del ciclo de mejora

Clase 1

\begin{tabular}{|l|l|l|l|}
\hline Actividad & Contenido & En qué consiste & T \\
\hline Presentación & - & $\begin{array}{l}\text { Saludar a los alumnos, encen- } \\
\text { der el ordenador y firmar en } \\
\text { Horfeus }\end{array}$ & $5^{\prime}$ \\
\hline $\begin{array}{l}\text { Repasar el } \\
\text { contexto }\end{array}$ & $\begin{array}{l}\text { Antecedentes } \\
\text { y objetivos del } \\
\text { nuevo bloque de } \\
\text { contenidos } \\
\text { Marco concep- } \\
\text { tual dentro del } \\
\text { programa de la } \\
\text { asignatura }\end{array}$ & $\begin{array}{l}\text { Exposición donde se explica } \\
\text { el nuevo bloque de conte- } \\
\text { nidos en el que se enmarca } \\
\text { esta lección (que es distinto } \\
\text { al de la anterior) y contextua- } \\
\text { lización en el desarrollo de la } \\
\text { asignatura }\end{array}$ & 5' \\
\hline
\end{tabular}

Jornadas de Formación e Innovación Docente del Profesorado | № 2 (2019) Esta obra se distribuye con la licencia Creative Commons Reconocimiento-NoComercial-SinObraDerivada Internacional (CC BY-NC-ND 4.0.) 


\begin{tabular}{|c|c|c|c|}
\hline $\begin{array}{l}\text { Explicar el } \\
\text { taller de } \\
\text { contenidos }\end{array}$ & $\begin{array}{l}\text { Instruccio- } \\
\text { nes del taller de } \\
\text { contenidos }\end{array}$ & $\begin{array}{l}\text { Explicar el desarrollo de la } \\
\text { actividad } \\
\text { Hacer los grupos de trabajo } \\
\text { Repartir las hojas con las ins- } \\
\text { trucciones y las series de } \\
\text { preguntas }\end{array}$ & $10^{\prime}$ \\
\hline $\begin{array}{l}\text { Estar pre- } \\
\text { sente en el } \\
\text { aula y a dis- } \\
\text { posición de } \\
\text { los grupos } \\
\text { de trabajo }\end{array}$ & $\begin{array}{l}\text { Contenidos con- } \\
\text { ceptuales de la } \\
\text { lección 8: Inter- } \\
\text { cambio radiante } \\
\text { en recintos con } \\
\text { medio participa- } \\
\text { tivo. Propiedades } \\
\text { radiantes de los } \\
\text { gases. Coeficiente } \\
\text { de absorción. Es- } \\
\text { pesor óptico. }\end{array}$ & $\begin{array}{l}\text { Los grupos irán dando res- } \\
\text { puesta a las preguntas inicial } \\
\text { y subordinadas, siguiendo las } \\
\text { instrucciones suministradas. } \\
\text { Mientras estaré presente en el } \\
\text { aula evitando posibles erro- } \\
\text { res de interpretación, guiando } \\
\text { el desarrollo intelectual, con- } \\
\text { trolando el desarrollo de la } \\
\text { actividad y, en definitiva es- } \\
\text { tando al servicio de los alum- } \\
\text { nos e interviniendo, si fuese } \\
\text { necesario. }\end{array}$ & $50^{\prime}$ \\
\hline $\begin{array}{l}\text { Conclusión y } \\
\text { despedida }\end{array}$ & & $\begin{array}{l}\text { Cierre de la actividad propor- } \\
\text { cionando la solución a las } \\
\text { respuestas y creando una sen- } \\
\text { sación de actividad concluida }\end{array}$ & $10^{\prime}$ \\
\hline
\end{tabular}

Clase 2

\begin{tabular}{|l|l|l|l|}
\hline Actividad & Contenido & En qué consiste & T \\
\hline Presentación & - & $\begin{array}{l}\text { Saludar a los alumnos, encen- } \\
\text { der el ordenador y firmar en } \\
\text { Horfeus }\end{array}$ & $5^{\prime}$ \\
\hline $\begin{array}{l}\text { Repasar } \\
\text { la lección } \\
\text { anterior }\end{array}$ & $\begin{array}{l}\text { Intercambio ra- } \\
\text { diante en at- } \\
\text { mósferas de } \\
\text { combustión }\end{array}$ & $\begin{array}{l}\text { Exposición dejando palabras } \\
\text { libres a ser completadas por } \\
\text { los alumnos }\end{array}$ & 10' \\
\hline
\end{tabular}

Jornadas de Formación e Innovación Docente del Profesorado | № 2 (2019) Esta obra se distribuye con la licencia Creative Commons 


\begin{tabular}{|c|c|c|c|}
\hline $\begin{array}{l}\text { Centrar a los } \\
\text { alumnos } \\
\text { Poner el } \\
\text { foco en el } \\
\text { problema } \\
\text { que quere- } \\
\text { mos resol- } \\
\text { ver con los } \\
\text { contenidos } \\
\text { de la lección }\end{array}$ & $\begin{array}{l}\text { Problema pre- } \\
\text { sentado en la en- } \\
\text { señanza virtual } \\
\text { con el nombre de } \\
\text { caso práctico no } 1 \\
\text { Contenido } \\
\text { conceptual/ } \\
\text { procedi-mental }\end{array}$ & $\begin{array}{l}\text { Recordar el caso práctico } 1 \\
\text { que ya resolvieron } \\
\text { Se trata de establecer un diá- } \\
\text { logo basado en preguntas } \\
\text { y respuestas con los alum- } \\
\text { nos para que identifiquen qué } \\
\text { es lo que nos hace falta co- } \\
\text { nocer para llegar a ser capa- } \\
\text { ces de evaluar el intercambio } \\
\text { radiante en el hogar. El caso } \\
\text { práctico ya resuelto se calculó } \\
\text { SIN transferencia de calor, } \\
\text { pero gracias a los contenidos } \\
\text { de este tema se van a poder } \\
\text { calcular con transferencia de } \\
\text { calor radiante. }\end{array}$ & $10^{\prime}$ \\
\hline $\begin{array}{l}\text { Hacer un es- } \\
\text { quema de la } \\
\text { lección }\end{array}$ & $\begin{array}{l}\text { Mapa de conte- } \\
\text { nido e índice de } \\
\text { la lección }\end{array}$ & $\begin{array}{l}\text { Presentar el mapa de conte- } \\
\text { nido y el índice de la lección } \\
\text { poniendo especial énfasis en } \\
\text { cómo los contenidos se ajus- } \\
\text { tan para lograr los objetivos } \\
\text { anteriormente identificados } \\
\text { en el caso práctico }\end{array}$ & $5^{\prime}$ \\
\hline $\begin{array}{l}\text { Enseñar me- } \\
\text { diante clase } \\
\text { magistral }\end{array}$ & $\begin{array}{l}\text { Contenidos con- } \\
\text { ceptuales y proce- } \\
\text { dimentales de la } \\
\text { Lección }\end{array}$ & $\begin{array}{l}\text { Clase magistral de } 26 \\
\text { diapositivas } \\
\text { (Hasta llama laminar y } \\
\text { turbulenta) }\end{array}$ & $45^{\prime}$ \\
\hline Despedida & - & $\begin{array}{l}\text { Es importante recordarles que } \\
\text { en la clase siguiente es nece- } \\
\text { sario traer ordenador (al me- } \\
\text { nos dos personas por grupo } \\
\text { de proyecto) }\end{array}$ & $5^{\prime}$ \\
\hline
\end{tabular}

Clase 3

\begin{tabular}{|l|l|l|l|}
\hline Actividad & Contenido & En qué consiste & T \\
\hline Presentación & - & $\begin{array}{l}\text { Saludar a los alumnos, encen- } \\
\text { der el ordenador y firmar en } \\
\text { Horfeus }\end{array}$ & $5^{\prime}$ \\
\hline
\end{tabular}

Jornadas de Formación e Innovación Docente del Profesorado | № 2 (2019) Esta obra se distribuye con la licencia Creative Commons 


\begin{tabular}{|c|c|c|c|}
\hline $\begin{array}{l}\text { Trabajar } \\
\text { personal- } \\
\text { mente en el } \\
\text { aula }\end{array}$ & $\begin{array}{l}\text { Entrenamiento } \\
\text { competencia }\end{array}$ & $\begin{array}{l}\text { En grupos de dos o tres, los } \\
\text { alumnos intentarán realizar el } \\
\text { caso práctico anterior. Dichos } \\
\text { grupos idealmente deberían } \\
\text { ser la mitad de un grupo de } \\
\text { proyecto de curso ya que es- } \\
\text { tos están compuestos por } 6 \\
\text { alumnos. } \\
\text { De esta forma también es } \\
\text { más probable que al me- } \\
\text { nos uno del grupo haga } \\
\text { muy bien la actividad y se } \\
\text { lo pueda transmitir al resto } \\
\text { posteriormente. }\end{array}$ & 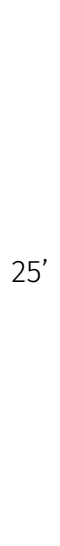 \\
\hline $\begin{array}{l}\text { Mostrar el } \\
\text { sistema de } \\
\text { ecuaciones } \\
\text { que debe- } \\
\text { rían haber } \\
\text { escrito }\end{array}$ & $\begin{array}{l}\text { Contenidos con- } \\
\text { ceptuales y proce- } \\
\text { dimentales de la } \\
\text { lección }\end{array}$ & $\begin{array}{l}\text { El profesor planteará en } \\
\text { clase el sistema de ecua- } \\
\text { ciones que, al haberse pro- } \\
\text { puesto de forma reducida, se } \\
\text { puede escribir en la pizarra. } \\
\text { Incluyendo las hipótesis, da- } \\
\text { tos necesarios de los que se } \\
\text { dispone o se pueden calcu- } \\
\text { lar y anticipando los que se } \\
\text { podrán calcular en el futuro } \\
\text { (p.ej. después de terminar la } \\
\text { lección siguiente en la que } \\
\text { aprenderán a calcular las pro- } \\
\text { piedades radiantes de gases } \\
\text { participativos) }\end{array}$ & 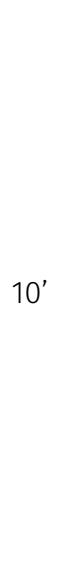 \\
\hline $\begin{array}{l}\text { Propo- } \\
\text { ner trabajo } \\
\text { para reali- } \\
\text { zar fuera del } \\
\text { aula }\end{array}$ & $\begin{array}{l}\text { Entrenamiento } \\
\text { competencia } \\
\text { mundo laboral } \\
\text { Contenido } \\
\text { procedimental }\end{array}$ & $\begin{array}{l}\text { Se propondrá a los alumnos } \\
\text { que repitan un ejercicio si- } \\
\text { milar al hecho en clase, pero } \\
\text { esta vez en grupos de } 6 \text { (sus } \\
\text { grupos naturales de proyecto } \\
\text { de curso creados con ante- } \\
\text { rioridad), reagrupando así los } \\
\text { grupos de } 2 \text { o } 3 \text {. Dicho ejer- } \\
\text { cicio consistirá en plantear y } \\
\text { resolver el sistema de ecua- } \\
\text { ciones, pero con el nivel de } \\
\text { detalle del caso original ( } 23 \\
\text { superficies y } 37 \text { volúmenes). }\end{array}$ & $10^{\prime}$ \\
\hline Despedida & & & $5^{\prime}$ \\
\hline
\end{tabular}

Jornadas de Formación e Innovación Docente del Profesorado | № 2 (2019) Esta obra se distribuye con la licencia Creative Commons 
Clase 4

\begin{tabular}{|c|c|c|c|}
\hline Actividad & Contenido & En qué consiste & $\mathrm{T}$ \\
\hline Presentación & - & $\begin{array}{l}\text { Saludar a los alumnos, encen- } \\
\text { der el ordenador y firmar en } \\
\text { Horfeus }\end{array}$ & $5^{\prime}$ \\
\hline $\begin{array}{l}\text { Revisar el } \\
\text { ejercicio } \\
\text { propuesto } \\
\text { Proporcio- } \\
\text { nar el re- } \\
\text { sultado, } \\
\text { guiar la ob- } \\
\text { tención de } \\
\text { resultados }\end{array}$ & $\begin{array}{l}\text { Contenido pro- } \\
\text { cedimental de la } \\
\text { lección }\end{array}$ & $\begin{array}{l}\text { Preguntas a los alumnos so- } \\
\text { bre el ejercicio propuesto al } \\
\text { final de la clase anterior, qué } \\
\text { han sido capaces de realizar } \\
\text { y qué no } \\
\text { Problemas encontrados } \\
\text { Exposición por parte de los } \\
\text { grupos de sus resultados }\end{array}$ & $30^{\prime}$ \\
\hline $\begin{array}{l}\text { Realizar } \\
\text { un análisis } \\
\text { Crítico }\end{array}$ & & $\begin{array}{l}\text { Interpretación de los resulta- } \\
\text { dos del trabajo anterior } \\
\text { Se plantean problemáticas } \\
\text { asociadas al tamaño del sis- } \\
\text { tema de ecuaciones que es } \\
\text { necesario resolver cada vez } \\
\text { que se modifique uno sólo de } \\
\text { los parámetros del horno o } \\
\text { generador }\end{array}$ & 20 \\
\hline $\begin{array}{l}\text { Presentar } \\
\text { las lecciones } \\
\text { siguientes }\end{array}$ & $\begin{array}{l}\text { Contenidos con- } \\
\text { ceptuales y proce- } \\
\text { dimentales de las } \\
\text { lecciones } 9,10 \text { y } 11\end{array}$ & $\begin{array}{l}\text { Mediante la proyección del } \\
\text { mapa de contenidos se mos- } \\
\text { trará y se pedirá la partici- } \\
\text { pación a los alumnos para } \\
\text { identificar la necesidad de las } \\
3 \text { lecciones siguientes, iden- } \\
\text { tificando lo que cada una } \\
\text { aporta, que a efectos prác- } \\
\text { ticos se muestra muy bien } \\
\text { ahora ya que como acaban de } \\
\text { terminar un problema pue- } \\
\text { den entender por qué nece- } \\
\text { sitan un método de evaluar } \\
\text { las propiedades de los ga- } \\
\text { ses, las áreas de intercambio } \\
\text { o resolver de forma simplifi- } \\
\text { cada el sistema de ecuacio- } \\
\text { nes tan complejo que acaban } \\
\text { de plantear }\end{array}$ & $10^{\prime}$ \\
\hline
\end{tabular}

Jornadas de Formación e Innovación Docente del Profesorado | № 2 (2019) Esta obra se distribuye con la licencia Creative Commons 


\begin{tabular}{|c|c|c|c|}
\hline $\begin{array}{l}\text { Plantear el } \\
\text { test de un } \\
\text { minuto o } \\
\text { cuestiona- } \\
\text { rio final en } \\
\text { el desarro- } \\
\text { llo del se- } \\
\text { gundo ciclo } \\
\text { de mejora }\end{array}$ & - & $\begin{array}{l}\text { Redacción de una carilla por } \\
\text { alumno en la que se recoja } \\
\text { la respuesta a las siguientes } \\
\text { preguntas ¿Qué es lo que más } \\
\text { te ha gustado de la lección? } \\
\text { ¿Qué es lo que te parece que } \\
\text { es más interesante para tu } \\
\text { vida profesional? ¿Te quedan } \\
\text { dudas o te gustaría profundi- } \\
\text { zar más en algunos concep- } \\
\text { tos? ¿Cuáles? }\end{array}$ & . \\
\hline Despedida & - & & $5^{\prime}$ \\
\hline
\end{tabular}

\section{Mapa de contenidos}

El mapa de contenidos que describe la lección desarrollada mediante la secuencia anterior es el representado en la figura siguiente. En el mismo se puede observar que la lección consiste en contenidos conceptuales y procedimentales, y que el objetivo de la misma es responder a la pregunta motivadora que aparece en la parte superior del esquema. No obstante, los contenidos conceptuales predominan sobre los procedimentales que se desarrollan en las lecciones sucesivas, tal y como se puede apreciar en la figura. No existen en la lección contenidos actitudinales.

Jornadas de Formación e Innovación Docente del Profesorado | № 2 (2019) Esta obra se distribuye con la licencia Creative Commons 


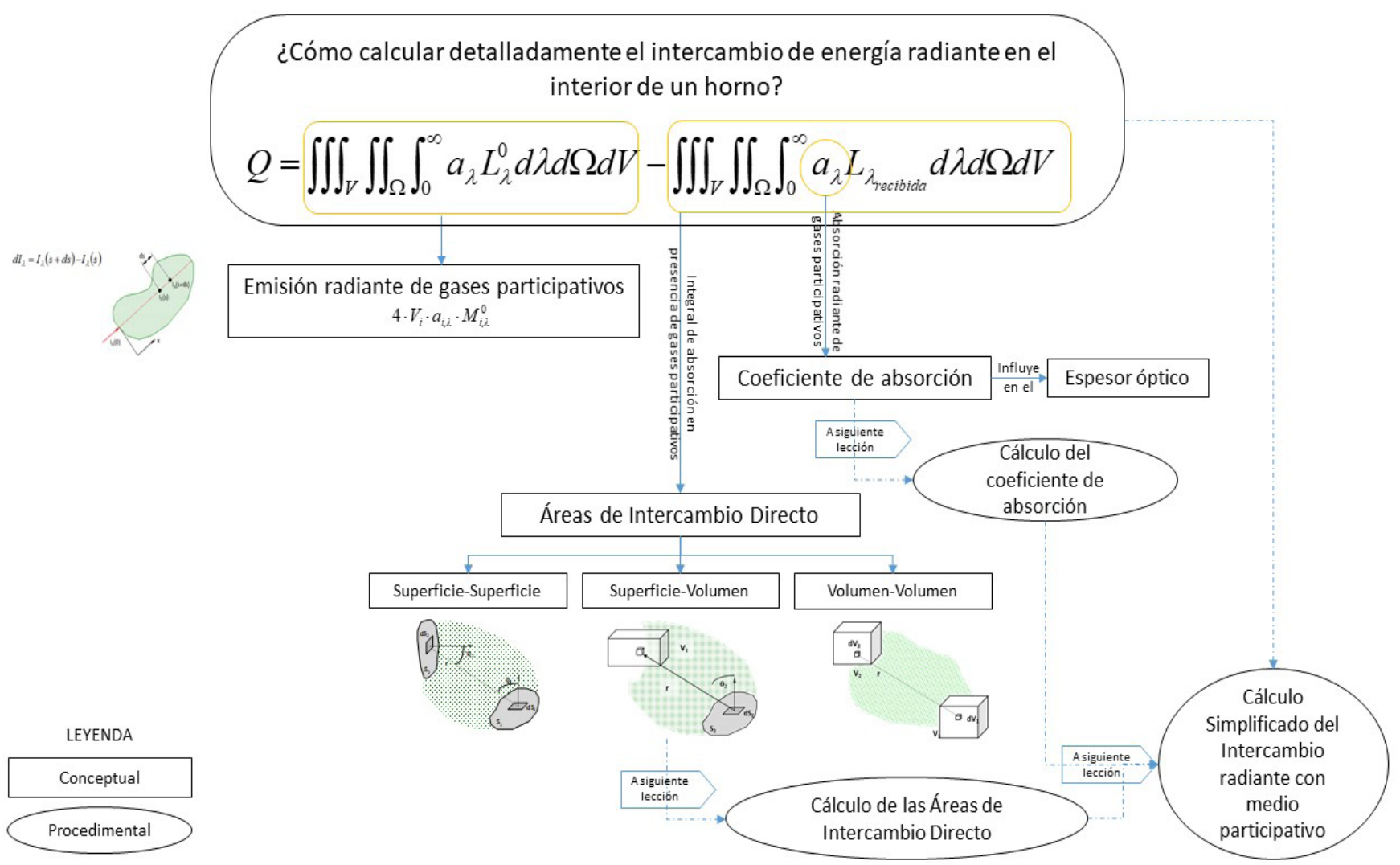

Figura 2. Mapa de contenidos

Jornadas de Formación e Innovación Docente del Profesorado I № 2 (2019)

(c) () E $\Theta$ Esta obra se distribuye con la licencia Creative Commons 


\section{Cuestionario de ideas previas}

Para conocer los esquemas mentales de los estudiantes, así como para hacer un seguimiento de la evolución de los mismos en relación con los problemas clave, se realizó el siguiente cuestionario:

Nombre o seudónimo:

1. ¿Para qué quiero o me sirve expresar los flujos de calor en función de las temperaturas? Razona brevemente tu respuesta

2. Cuando entras en una piscina puedes haber notado que la superficie del agua está más caliente, ¿crees que está relacionado con la radiación? ¿por qué?

3. ¿Son todos los medios iguales en radiación? Haz una pequeña lista (de 2 o 3 puntos) de similitudes y diferencias que afecten a la radiación en las siguientes parejas de recintos:

a) Un aula y el interior de una caldera de combustión

\begin{tabular}{|l|l|}
\hline Diferencias & Similitudes \\
\hline & \\
\hline & \\
\hline
\end{tabular}

b) Un aula y la atmósfera

\begin{tabular}{|l|l|}
\hline Diferencias & Similitudes \\
\hline & \\
\hline & \\
\hline
\end{tabular}

4. En un recinto de los anteriores, ¿es importante la radiación que llega a una superficie proveniente de otras para poder decir el flujo de calor neto emitido por dicha superficie? Razona tu respuesta

5. Nombra componentes de una llama o de los gases producidos en la misma que puedan absorber radiación 
6. ¿Crees que las partículas presentes en una llama o en los gases absorben radiación? ¿por qué?

Una novedad en el desarrollo del mismo es que se ha creado usando preguntas que pueden ser usadas en el desarrollo del taller de contenidos como señala Finkel (2000), y que está previsto en la primera clase de aplicación del ciclo de mejora. En este caso, el cuestionario inicial que se pasó a los alumnos consistió en las 4 primeras preguntas, en el taller se utilizaron de la 1 a la 5. En el cuestionario final se plantearon las seis.

\section{Aplicación de los ciclos de mejora de aula}

\section{Valoración de las sesiones de aplicación del ciclo de mejora}

La valoración global de la aplicación de las sesiones de los ciclos de mejora es muy positiva. Los alumnos se sienten más involucrados en su aprendizaje, sobre todo cuando se aplican nuevas metodologías como el "taller de contenidos", sienten curiosidad y se percibe que agradecen tomar las riendas de su propio aprendizaje. El cuestionario inicial les hace valorar de forma más objetiva su nivel de conocimientos y, al comparar con sus compañeros o por los comentarios del profesor pueden re-elaborar sus estructuras mentales relativas a los conceptos tratados en la lección. El desarrollo de las actividades es fluido, pese a que en algunos casos se preveía el riesgo de no avanzar por razonamientos equivocados que requiriesen el empleo de mucho tiempo para reconducir la situación. Si bien es cierto que la planificación de las clases debería hacerse dedicando más tiempo a cada actividad, porque normalmente la clase acababa muy justa o con un cierto retraso. 


\section{Evaluación del aprendizaje de los estudiantes}

Siguiendo las indicaciones de la metodología de análisis del cuestionario y basándonos en las publicaciones de aplicación de ciclos de mejora previos, se han identificado mediante un número o código ID que se ha usado para mostrar los resultados manteniendo el anonimato de los estudiantes en las tablas.

Las cuestiones, los escalones de aprendizaje y las barreras a superar que se han identificado a a partir de las respuestas de los alumnos se detallan a continuación:

Cuestión 1: ¿Para qué quiero o me sirve expresar los flujos de calor en función de las temperaturas? Razona brevemente tu respuesta

Los escalones de aprendizaje identificados según el análisis de la respuesta de los alumnos son los siguientes:

A. Sin responder

B. Para calcular los flujos de calor o mencionar la relación entre ambas o vincular la respuesta con la discretización volumétrica.

C. Para calcular las temperaturas o calcular el campo de temperaturas

D. Para poder calcular las temperaturas mediante balances de energía volumétricos

Las tabicas de la escalera de aprendizaje identificadas son las siguientes:

A->B Se comprende que existe la necesidad, pero se menciona la pregunta en la respuesta, el alumno no se ha planteado con profundidad ni sabe expresar la necesidad del objetivo inicial de la lección

$\mathrm{B} \rightarrow \mathrm{C}$ El alumno entiende que el verdadero objetivo que está buscando es conocer el campo de temperaturas, pero no conoce la forma de conseguirlo

C->D El alumno comprende la necesidad de expresar los flujos en función de las temperaturas para calcular 
éstas planteando un balance de energía, cuyas incógnitas serán precisamente las temperaturas que, una vez conocidas, servirán para calcular los flujos de calor.

Cuestión 2: Cuando entras en una piscina puedes haber notado que la superficie del agua está más caliente, ¿crees que está relacionado con la radiación? ¿por qué?

Los escalones de aprendizaje identificados según el análisis de la respuesta de los alumnos son los siguientes:

A. Sí cree que existe relación pero no sabe decir el porqué o bien entra en contradicciones

B. Si cree que existe relación pero cae en errores como que no existe radiación en líquidos, o bien cree que los líquidos son impermeables al paso de la radiación.

C. Sí cree que existe relación e incluye otros mecanismos en el razonamiento, como por ejemplo la transferencia por convección hacia el fondo. En ocasiones incluyen una estimación del porcentaje de radiación absorbida en la capa superficial.

D. Sí cree que existe relación e incluye otros mecanismos en el razonamiento, explicita que el agua es un medio participativo por el alto contenido en moléculas dipolares como son las del H2O.

Las tabicas de la escalera de aprendizaje son las siguientes:

A->B Se comprende el origen del fenómeno, si bien se desconocen algunas características básicas de los mecanismos

B $>$ C El alumno entiende los fundamentos del fenómeno y algunas características básicas, incluso el orden de magnitud de las variables

C $\rightarrow$ D El alumno entiende los fundamentos del fenómeno y sus características básicas además de conocer la denominación y las particularidades moleculares que provocan que un medio sea participativo 
Cuestión 3: ¿Son todos los medios iguales en radiación? Haz una pequeña lista (de 2 o 3 puntos) de similitudes y diferencias que afecten a la radiación en las siguientes parejas de recintos:

a) Un aula y el interior de una caldera de combustión

b) Un aula y la atmósfera

Los escalones de aprendizaje identificados según el análisis de la respuesta de los alumnos son los siguientes:

A. No responde o bien lo hace con muchas imprecisiones en los pocos puntos incluidos

B. Responde con pocas imprecisiones pero lo hace mencionando pocos puntos de diferencias y similitudes, los mismos no son determinantes

C. Responde con pocas o nulas imprecisiones, mencionando bastantes puntos de diferencias y similitudes y los mismos son importantes

D. Responde sin imprecisiones, mencionando tres diferencias y similitudes en cada caso, y las mismas son fundamentales

Las tabicas de la escalera de aprendizaje son las siguientes:

A->B Se conoce algunas similitudes y diferencias del mecanismo radiante y el grado de participación de gas en el recinto, si bien se desconocen las más determinantes

$\mathrm{B}->\mathrm{C}$ El alumno conoce y comprende el origen de algunas de las similitudes y diferencias del mecanismo radiante y el concepto de espesor óptico no le es ajeno aunque no lo abarca completamente.

C $>$ D El alumno conoce y comprende el origen de las similitudes y diferencias del mecanismo radiante y abarca completamente el concepto de espesor óptico.

Cuestión 4: En un recinto de los anteriores, ¿es importante la radiación que llega a una superficie proveniente de otras para poder decir el flujo de calor neto emitido por dicha superficie? Razona tu respuesta 
Los escalones de aprendizaje identificados según el análisis de la respuesta de los alumnos son los siguientes:

A. No responde o responde negativamente

B. Responde afirmativamente pero no lo justifica

C. Responde afirmativamente, pero la justificación no es correcta del todo o es correcta pero difusa

D. Responde afirmativamente y la justificación es completamente correcta

Las tabicas de la escalera de aprendizaje son las siguientes:

A->B Se comprende que la radiación está siempre influenciada por el resto de superficies pero no se sabe el porqué

B->C Se comprende que la radiación está siempre influenciada por el resto de superficies y se conoce el motivo de lo anterior de forma difusa

C->D Se comprende que la radiación está siempre influenciada por el resto de superficies y se conoce detalladamente el motivo de lo anterior

El resultado del progreso global de los alumnos en base a sus respuestas a todas las cuestiones puede verse en la siguiente tabla:

Tabla 2. Resultados de los cuestionarios inicial y final clasificados por cuestiones.

\begin{tabular}{|c|c|c|c|c|c|c|c|c|}
\hline & \multicolumn{4}{|c|}{ Inicial } & \multicolumn{4}{c|}{ Final } \\
\hline & $\mathrm{A}$ & $\mathrm{B}$ & $\mathrm{C}$ & $\mathrm{D}$ & $\mathrm{A}$ & $\mathrm{B}$ & $\mathrm{C}$ & $\mathrm{D}$ \\
\hline Cuestión 1 & $17 \%$ & $25 \%$ & $42 \%$ & $17 \%$ & $0 \%$ & $17 \%$ & $58 \%$ & $25 \%$ \\
\hline Cuestión 2 & $25 \%$ & $50 \%$ & $17 \%$ & $8 \%$ & $8 \%$ & $17 \%$ & $33 \%$ & $42 \%$ \\
\hline Cuestión 3 & $25 \%$ & $50 \%$ & $25 \%$ & $0 \%$ & $0 \%$ & $17 \%$ & $33 \%$ & $50 \%$ \\
\hline Cuestión 4 & $42 \%$ & $50 \%$ & $8 \%$ & $0 \%$ & $17 \%$ & $25 \%$ & $33 \%$ & $25 \%$ \\
\hline
\end{tabular}

Como puede verse en la situación inicial los alumnos estaban mayoritariamente en el escalón B, y entre el A y 
el B estaban casi el 75\% del total. En la situación final el $75 \%$ de los alumnos están entre el escalón C y el D, siendo los porcentajes de estas dos categorías el $40 \%$ y el $35 \%$ respectivamente.

\section{Conclusiones}

La aplicación del programa de mejora de aula en la asignatura ha puesto de manifiesto la importancia de los siguientes aspectos:

\section{Contenidos de la asignatura}

La realización del mapa de contenidos se ha demostrado como una herramienta muy útil para dotar de lógica y poder razonar los contenidos presentes en el temario de una asignatura. En primer lugar, distinguiendo entre contenidos conceptuales, procedimentales y actitudinales. Lo anterior tiene varios fines:

- Equilibrar los contenidos conceptuales y procedimentales

- Estructurar el temario de un bloque, viendo las relaciones entre los contenidos y facilitando así el "camino" de aprendizaje de los alumnos.

- Estructurar el contenido de la asignatura completa, y dar a entender mejor a los alumnos las relaciones existentes entre las distintas lecciones o bloques temáticos de la asignatura.

- Estructurar gráficamente los contenidos, marcando sus relaciones y representando las jerarquías existentes entre los mismos.

- Reflexionar sobre la mejor forma de impartir los contenidos para que el aprendizaje de los mismos por parte de los alumnos sea más llevadero, sin tener que aparcar demasiadas cosas hasta haber 
aprendido más de lo que se está explicando en el tema actual.

\section{Metodología docente}

La sentencia "damos clase intentando responder a preguntas que los alumnos no se han hecho" (Bain, 2007) ha sido reveladora para el autor porque, al pensarla, y luego tratar de aplicarla en la práctica hemos percibido que es totalmente cierta. La mayor modificación metodológica ha consistido en adelantar los problemas que vamos a resolver al principio de la lección, para que los alumnos sientan curiosidad, se hagan preguntas, y se orienten o tengan un punto de referencia sobre lo que queremos conseguir. Estos problemas son muchas veces sustitutos o, mejor dicho, complementos perfectos, de la pregunta motivadora para los alumnos. Todas las actividades están articuladas y giran alrededor de este problema central.

El uso y aplicación en clase del "taller de conceptos" es algo que ha resultado muy satisfactorio, el mismo requiere una preparación importante pues las preguntas que se reparten a los alumnos para realizar durante el tiempo de la clase deben seguir un orden y tener unos objetivos muy concretos para que sirvan para guiar el proceso de aprendizaje. No obstante, es muy útil adicionalmente para la evaluación de los alumnos y para organizar el mapa de contenidos de la lección.

\section{Aprendizaje de los alumnos y evaluación}

Como consecuencia de la aplicación del programa de mejora docente he aprendido a realizar la evaluación de los modelos mentales de los alumnos, sus conocimientos previos y cómo los mismos están estructurados; esto me ha llevado a pensar en los contenidos gracias a la 


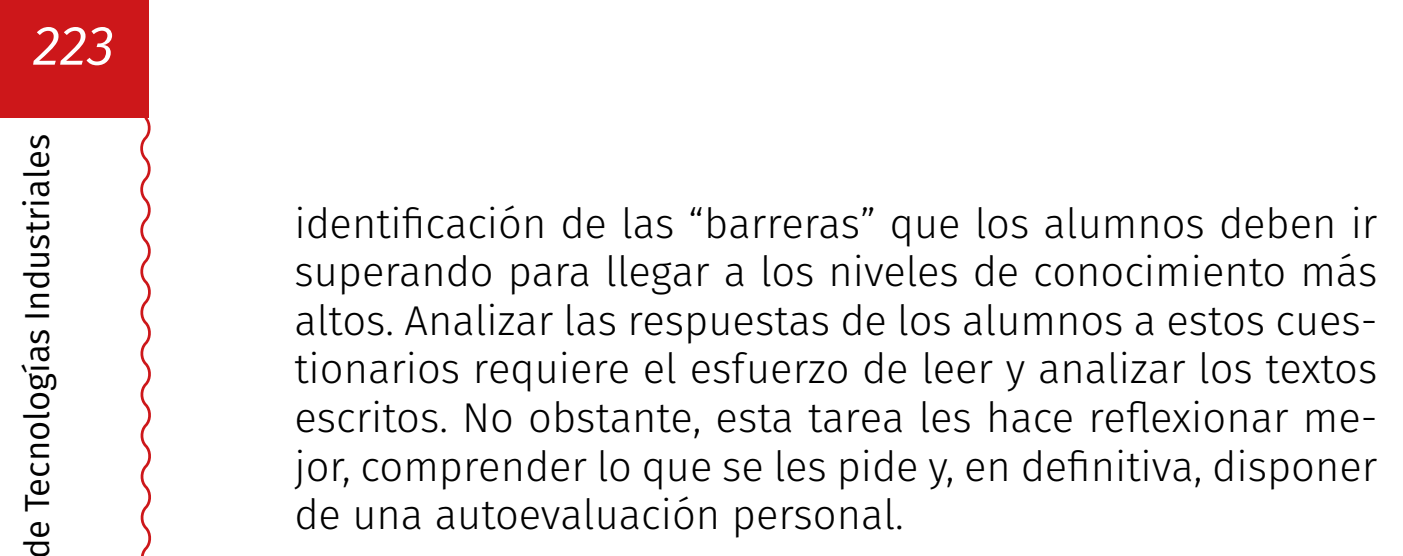
altos. Analizar las respuestas de los alumnos a estos cuestionarios requiere el esfuerzo de leer y analizar los textos escritos. No obstante, esta tarea les hace reflexionar mejor, comprender lo que se les pide y, en definitiva, disponer de una autoevaluación personal. 


\section{Referencias Bibliográficas}

Barbadilla-Martín E. (2017). Acercando la realidad de gestiōn de proyectos en ingeniería, Jornadas de formación e innovacion docente, 217-228. Sevilla: I.C.E. Universidad de Sevilla.

Bain, K. (2007). Lo que hacen los mejores profesores universitarios. Valencia: Publicaciones de la Universidad de Valencia.

Beltrán, A.M. (2017). Mejora docente en primer curso de ingeniería, Jornadas de formación e innovación docente, 279-286. Sevilla: I.C.E. Universidad de Sevilla.

Finkel, D. (2000). Dar clase con la boca cerrada. Valencia: Publicaciones de la Universidad de Valencia.

Leñero, J.A. (2016). Sobre la importancia de plantear, resolver y debatir problemas desde el primer día de clase en asignaturas de ingeniería, Jornadas docencia universitaria, 622-631. Sevilla: I.C.E. Universidad de Sevilla.

Pérez J.A. (2016). Uso de una herramienta de simulación de comunicaciones satélite, Jornadas docencia universitaria, 746-757. Sevilla: I.C.E. Universidad de Sevilla.

Pérez, P. (2016). Aplicación de un nuevo modelo didáctico en ingeniería de organización, Jornadas docencia universitaria, 230-240. Sevilla: I.C.E. Universidad de Sevilla.

Rivero, A. y Porlán, R. (2017). La evaluación en la enseñanza universitaria. En R. Porlán (Coord.), Enseñanza universitaria. Como mejorarla (pp. 73-91). Madrid: Morata.

Jornadas de Formación e Innovación Docente del Profesorado | № 2 (2019) Esta obra se distribuye con la licencia Creative Commons 